Ann. Biol. anim. Bioch. Biophys., I968, 8 (2), 273-280.

\title{
UTILISATION DIGESTIVE, CINÉTIQUE DE L'ABSORPTION ET EFFICACITÉ DE LA RÉTENTION DU PHOSPHORE PHYTIQUE CHEZ LE PORC
}

\author{
L. GUÉGUEN, P. BESANÇON et A. RÉRAT \\ avec la collaboration technique de Pierrette Camus et Colette Katz \\ Station centrale de Recherches de Nutrition, \\ Station de Recherches sur l'Élevage des Porcs, \\ Centre national de Recherches zootechniques, 78 - Jouy-en-Josas \\ Institut national de la Recherche agronomique
}

\section{SOMMAIRE}

L'expérience a porté sur $\mathbf{I} 7$ porcs en croissance, ayant reçu une dose orale de ${ }^{32} \mathrm{P}$, soit sous forme de phosphate disodique, soit sous forme de son de blé irradié. Les fèces et l'urine ont été collectées pendant 6 jours après le repas marqué et des prélèvements de sang ont été effectués pour l'étude cinétique de l'absorption intestinale.

Le coefficient d'utilisation digestive réelle du phosphore a atteint en moyenne $7 \mathrm{r}, 0 \mathrm{p}$. roo pour le phosphate disodique et $37,5 \mathrm{p}$. Ioo pour le son de blé. L'hydrolyse des phytates semble se produire principalement dans l'estomac.

Le maximum de radioactivité plasmatique a été obtenu au bout d'une heure pour le phosphate disodique et au bout de 4 heures pour le son de blé, dont le phosphore est beaucoup plus lentement absorbé. La valeur du maximum de radioactivité plasmatique (p. Ioo de la dose administrée) ne peut être employée comme critère de l'intensité de l'absorption du phosphore.

L'efficacité de la rétention du phosphore absorbé est plus faible pour le son de blé (6I,3 p. 100) que pour le phosphate disodique $(76, \mathrm{I}$ p. I00). L'interprétation de cette différence est basée sur l'hypothèse selon laquelle la simultanéité de l'absorption du phosphore et de l'absorption du calcium serait un facteur favorable à la rétention osseuse maximale du phosphore absorbé.

\section{INTRODUCTION}

I.e problème de 1'utilisation par les animaux du phosphore phytique contenu dans les aliments d'origine végétale n'est pas encore bien résolu. S'il est maintenant admis que les Ruminants, grâce aux phytases bactériennes du rumen, sont capables d'utiliser normalement cette forme de phosphore, par contre, de grandes divergences de vues existent encore entre auteurs pour ce qui concerne les Monogastriques.

Mis à part les expériences de MoORE et TyLER (I955) sur le Porc, la plupart des 
travaux concernant le phosphore phytique, récemment revus par NELson (I967), ont porté sur les volailles. De nombreux auteurs, cités par WALDROUP et al. (I965), ont longtemps émis l'opinion selon laquelle le phosphore phytique permettait d'assurer à l'animal une croissance corporelle normale mais était incapable d'assurer une bonne minéralisation de l'os. Ainsi, selon Sнонц (I939) et Singsen (I948), le phosphore des phytates peut être absorbé directement, sans hydrolyse, et être utilisé pour satisfaire les besoins de croissance ; cependant, la molécule de phytine doit être rompue afin que le phosphore soit utilisable par l'os. Il est surprenant qu'il puisse ainsi exister, après le passage du phosphore dans le sang, une discrimination en fonction de son origine pour l'utilisation métabolique de l'élément. En fait, des travaux plus récents de Temperton et CAssidy (I964), de Singsen et al. (I950) et de WALDROuP et al., (I967) démontrent que le phosphore doit être libéré de la molécule de phytine avant d'être absorbé et qu'il peut ensuite participer à toutes les réactions nécessitant du phosphore.

Nous avons voulu préciser ce phénomène chez le Porc en comparant le métabolisme du phosphore (en majorité phytique) du son de blé, d'une part, et du phosphore du phosphate disodique d'autre part. Nous avons comparé ces deux produits en étudiant les différences d'utilisation digestive réelle et d'efficacité de la rétention du phosphore. Puis, nous avons tenté d'interpréter les différences observées, à l'aide des cinétiques comparées de l'absorption intestinale du phosphore.

\section{MATÉRIEL ET MÉTHODES}

L'expérience a porté sur I 7 porcs mâles de race Large White, pesant de 30 à $50 \mathrm{~kg}$ et maintenus en cages à métabolisme. Le régime alimentaire, à base de céréales et de tourteaux, était distribué en trois repas de 500 à $600 \mathrm{~g}$ par jour. L'aliment contenait $9 \mathrm{~g}$ de $\mathrm{Ca}, 8 \mathrm{~g}$ de $\mathrm{P}$ total, et $\mathrm{I}$ o0o UI de vitamine $\mathrm{D}_{3}$ par $\mathrm{kg}$ de matière sèche.

La méthode des repas marqués a été utilisée, qui consiste à fournir à l'animal une dose de ${ }^{32} \mathrm{P}$ intimement mélangée au repas du matin, et à mesurer durant 6 jours l'excrétion de radioactivité dans les fèces et dans l'urine. La dose de ${ }^{32} \mathrm{P}$ (environ $200 \mu \mathrm{Ci}$ ) a été administrée, soit sous la forme de ${ }^{32} \mathrm{PO}_{4} \mathrm{HNa}_{2}$ en solution, soit sous la forme de 5 à $10 \mathrm{~g}$ de son de blé irradié. Une courte activation aux neutrons dans un réacteur nucléaire permet de marquer intimement le phosphore dans sa molécule organique, sans que les liaisons chimiques soient sensiblement modifiées par effet Szilard-Chalmers. Le dosage du phosphore phytique dans le son avant et après irradiation, à l'aide de la méthode de HoLT (I955), a montré que la proportion de phosphore phytique par rapport au phosphore total demeurait de $85 \mathrm{p}$. roo environ. D'autre part, nous avons vérifié que ${ }^{32} \mathrm{P}$ était le seul radionuclide détectable au moment de l'emploi (au moins 8 jours après l'irradiation).

Deux essais successifs, séparés par un intervalle de temps de ro jours, ont été effectués sur le même animal. Ainsi, I 2 essais comportaient une administration orale de ${ }^{32} \mathrm{PO}_{4} \mathrm{HNa}_{2}$, incluant les 4 essais d'une expérience antérieure (GUEGUEN et RÉRAT, 1967), I6 essais portaient sur du son de blé irradié et 6 essais sur un extrait trichloracétique de son de blé irradié (l'extraction par l'acide trichloracétique à $20 \mathrm{p}$. Ioo permettant d'administrer le phosphore phytique sous forme liquide).

Les animaux étant munis de cathéters placés en permanence dans les deux veines jugulaires, il a été possible d'étudier, dans environ la moitié des essais effectués, les variations de la radioactivité plasmatique en fonction du temps écoulé après le repas marqué. Ainsi, ro à 12 prélèvements de sang ont été faits sur chaque animal durant les huit heures suivant l'administration orale de ${ }^{32} \mathrm{P}$.

Le sang a été immédiatement centryifugé pour en séparer le plasma. Le phosphore total a été dosé dans le plasma, l'urine et les fèces, à l'aide de la méthode colorimétrique au métavanadate d'ammonium, après minéralisation par voie sèche. Le calcium a été dosé dans le plasma par photométrie de flamme. Les mesures de radioactivité ont été faites sur les solutions minéralisées à l'aide d'un compteur Geiger-Müller à fenêtre mince associé à un passeur automatique d'échantillons. Les valeurs obtenues pour la radioactivité plasmatique ont été rapportées à $100 \mathrm{mg}$ de calcium, soit environ 1 litre de plasma. Le dosage du calcium dans chaque prélèvement permet une estimation indirecte précise du volume de vrai plasma prélevé. 


\section{RÉSULTATS}

\section{Utilisation digestive et métabolique du phosphore}

Sachant qu'environ 4 p. roo d'une dose de ${ }^{32} \mathrm{P}$ injectée (ou absorbée) se retrouvent dans les fèces au bout de 6 jours (GUEGUEN et RÉRAT, I967), nous considérons que le coefficient d'utilisation digestive réelle $\left(\mathrm{CUD}_{r}\right)$ de ${ }^{32} \mathrm{P}$ ingéré est approximativement égal au pourcentage de la dose ingérée non éliminé dans les fèces, divisé par 0,96 ; ceci correspond à la correction nécessitée par l'excrétion fécale de radioactivité endogène, selon la technique des bilans comparés (Comar et al., I953). Bien entendu, le CUD $r$ ainsi obtenu ne concerne pas le phosphore de la ration totale mais seulement le phosphore de la source marquée.

Les résultats obtenus sont résumés dans le tableau I. Le pourcentage de la dose ingérée excrété dans l'urine est en moyenne de I4 p. Ioo et varie peu d'un traitement à l'autre. En revanche, l'utilisation digestive et la rétention de ${ }^{32} \mathrm{P}$ (exprimée par rapport à la dose ingérée ou par rapport à la fraction absorbée) sont très significativement plus faibles (à $P<0,0 r$ ) dans le cas du son de blé. Non seulement le phosphore du son de blé est moins bien absorbé que le phosphore du phosphate disodique, mais encore la fraction absorbée est moins bien retenue (6I p. Ioo au lieu de 76 p. I0o). Ainsi, par rapport au phosphore d'une source soluble, le phosphore phytique ingéré est approximativement deux fois moins bien absorbé et moins bien retenu.

TABLEAU I

Influence de la nature de l'apport alimentaire sur le métabolisme du phosphore

\begin{tabular}{|c|c|c|c|c|}
\hline & $\begin{array}{l}{ }^{32} \mathrm{P} \text { absorbé } \\
\text { p. } 100 \text { ingéré }\end{array}$ & $\begin{array}{l}{ }^{32} \mathrm{P} \text { retenu } \\
\text { p. } 100 \text { ingéré }\end{array}$ & $\begin{array}{l}\text { a2P retenu } \\
\text { p. } 100 \text { absorbé }\end{array}$ & $\begin{array}{l}\text { 32P urinaire } \\
\text { p. } 100 \text { ingéré }\end{array}$ \\
\hline 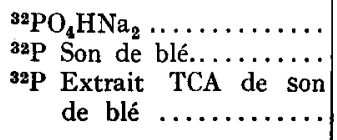 & $\begin{array}{l}71,0 \pm 4,8^{*} \\
37,5 \pm 2,5 \\
28,0 \pm 2,1\end{array}$ & $\begin{array}{l}54,2 \pm 4,4 \\
23,0 \pm 2,8 \\
13,0 \pm 2,7\end{array}$ & $\begin{array}{l}76,1 \pm 2,5 \\
61,3 \pm 4,5 \\
46,4 \pm 7,3\end{array}$ & $\begin{array}{r}14,0 \pm 1,6 \\
13,1 \pm 1,1 \\
14,0 \pm 1,1\end{array}$ \\
\hline
\end{tabular}

* Erreur-type.

L'emploi d'un extrait TCA de son de blé avait principalement pour but de fournir à l'animal du phosphore phytique sous forme liquide afin de diminuer la durée de séjour dans l'estomac (AUFFRAY et $a l$., I967). Les résultats obtenus pour l'absorption et la rétention sont dans ce cas inférieurs aux résultats concernant le son sous forme solide ; cependant, les différences ne sont pas significatives (à $P<0,05$ )

\section{Cinétique de l'absorption intestinale de ${ }^{32} P$}

Sur les courbes de la figure $I$ sont représentées les valeurs moyennes obtenues dans chaque traitement pour la radioactivité plasmatique exprimée par litre de plasma en pourcentage de la dose administrée. Pour le phosphate disodique, le son 
de blé et l'extrait TCA de son de blé la radioactivité apparaît dans le plasma respectivement Io, 30 et $15 \mathrm{mn}$ après le début du repas (qui dure de 5 à Io $\mathrm{mn}$ ) et les maxima sont enregistrés respectivement au bout de $I h, 4 h$ et $2 \mathrm{~h}$.

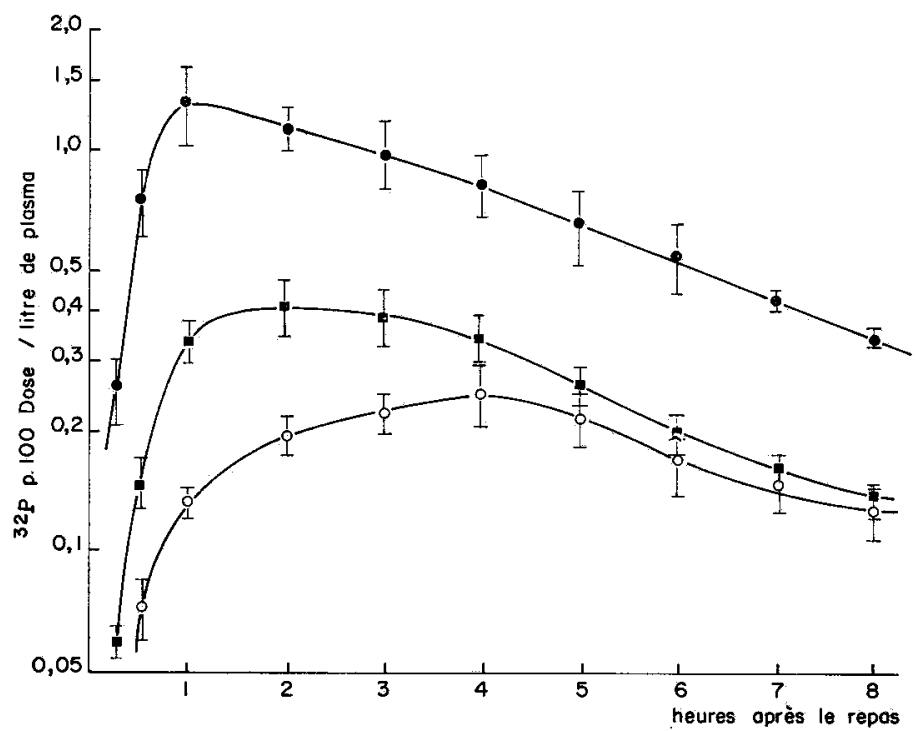

FIG. I. - Variations de la radioactivité plasmatique après administration orale de diverses sources marquées de phosphore

- ${ }^{32} \mathrm{PO}_{4} \mathrm{HNa}_{2}$

o ${ }^{32} \mathrm{P}$ : Son de blé

- ${ }^{32} \mathrm{P}$ : Extrait TCA de son de blé

Ainsi, le phosphore phytique du son de blé est absorbé beaucoup plus lentement que le phosphore du phosphate disodique ; aussi, il n'est pas possible d'utiliser dans ce cas le modèle décrit précédemment (GUEGUEN et RéRAT, I967) pour calculer la vitesse de l'absorption. D'autre part, lorsque l'allure des courbes cinétiques d'absorption varie, ni la valeur du maximum obtenu dans le plasma, ni la surface délimitée par la courbe, ne peuvent être proportionnelles au CUD $r$ correspondant. Par exemple, pour un CUD égal ou plus faible, la valeur du maximum plasmatique est plus élevée lorsque le phosphore phytique est ingéré sous forme liquide, c'està-dire lorsque l'absorption intestinale est plus rapide.

\section{DISCUSSION}

I. Utilisation digestive $d u$ phosphore phytique

Par rapport au phosphore du phosphate disodique, le phosphore du son de blé est environ deux fois moins bien absorbé. Ce résultat contribue à confirmer le facteur de correction de 0,5 que l'on emploie généralement pour évaluer chez le Porc l'apport de phosphore par les céréales. 
Si l'on tient compte des échanges éventuels pouvant se produire entre ${ }^{32} \mathrm{P}$ du son et le phosphore minéral stable de la lumière de l'intestin, échanges signalés par MOORE et TyLER (I955 b), puis par NiLSON (1967), une partie du ${ }^{32} \mathrm{P}$ absorbé le serait sous une forme chimique différente de la forme d'apport, et probablement plus utilisable. La valeur moyenne de $37,5 \mathrm{p}$. Ioo que nous avons obtenue peut donc être considérée comme étant une valeur maximale du CUD réel du phosphore du son de blé.

La diminution (bien que peu significative) du CUD du phosphore phytique lorsqu'il est administré sous la forme d'un extrait trichloracétique, pourrait s'expliquer par une interruption de 1'hydrolyse, due au passage plus rapide des molécules de phytine dans l'intestin. En effet, si l'on applique à 1'animal les résultats obtenus in vitro par HIL, et TYLER ( $\mathrm{I}_{954} b$ ), montrant que les phytases alimentaires ont leur maximum d'activité à $\mathrm{pH} 5$ et qu'elles sont irréversiblement inhibées à un $\mathrm{pH}$ inférieur à 3 , on peut conclure que les phytases fournies par l'aliment sont inactivées dans l'estomac et n'agissent plus dans l'intestin.

Des phytases microbiennes pourraient aussi intervenir, principalement dans le cæcum, mais il est vraisemblable que le phosphore ainsi libéré ne peut plus être absorbé à ce niveau. Des résultats préliminaires obtenus sur deux porcs nous ont montré qư'après administration dans le cæcum (à l'aide d'une canule permanente) d'une dose de ${ }^{32} \mathrm{PO}_{4} \mathrm{HNa}_{2}$ en solution, aucune trace de radioactivité n'était décelable, ni dans le sang, ni dans l'urine ; toute la dose administrée était excrétée dans les fèces (données non publiées).

L'hydrolyse des phytates, nécessaire à l'absorption du phosphore, se produirait donc principalement dans les zones de l'estomac où le $\mathrm{pH}$ n'est pas encore inférieur à 3 .

\section{Cinétique de l'absorption intestinale du phosphore}

L'absorption plus lente du phosphore du son administré sous forme solide serait simplement due au séjour prolongé dans l'estomac, d'où il est progressivement évacué. L'allure des courbes cinétiques d'absorption n'étant pas toujours la même, il n'est pas possible, comme nous l'avons déjà souligné (GUEGUEN et RÉRAT, I963), d'utiliser, soit la valeur du maximum de radioactivité plasmatique, soit la valeur obtenue dans le plasma au bout d'un temps déterminé, comme critères de l'efficacité de 1'absorption. Même si les vitesses de sortie du radio-isotope vers l'urine et vers l'os sont identiques, les valeurs des maxima observés ne peuvent être proportionnelles aux CUD $r$ obtenus. Les tests de radioactivité plasmatique utilisés chez 1'Homme pour évaluer l'absorption du calcium (CANigGia et GenNari I965. Avioli et al. I965) et du phosphore (CANiggra et al., I966) ne peuvent donc être valables que si les vitesses de transit digestif et de libération de l'élément devant être absorbé sont constantes en fonction du sujet ou du régime alimentaire.

\section{Rétention du phosphore absorbé}

Le phosphore absorbé en provenance du son de blé est moins bien retenu que le phosphore du phosphate disodique. Ainsi, environ $20 \mathrm{p}$. Ioo de la quantité de ${ }^{32} \mathrm{P}$ absorbée est éliminée par l'urine dans le cas du phosphate disodique contre 35 à 50 p. roo dans le cas du son de blé. Ces résultats sont en accord avec les observations 
déjà faites par plusieurs auteurs concernant la mauvaise rétention du phosphore phytique absorbé.

Ayant rejeté, pour les raisons déjà développées, l'hypothèse d'une absorption intestinale directe de la phytine dont le phosphore ne peut ensuite participer à toutes les réactions, il faut essayer de fournir à ce phénomène une autre interprétation, basée sur les différences de vitesse d'absorption. Paradoxalement, le phosphore est moins bien retenu lorsqu'il est absorbé lentement. Une constatation du même ordre avait déjà été faite (GUEGUEN et RÉRAT, 1963) pour le phosphore d'un phosphate alumino-ferrique, lentement absorbé et également mal retenu. La même cause pourrait être attribuée à la mauvaise rétention du phosphore des méta- et pyrophosphates étudiés par Ammerman et al. (1957) et qui, par rapport aux orthophosphates, provoquent un accroissement des pertes endogènes fécales de phosphore; en effet, dans ce cas, une hydrolyse préalable serait nécessaire et retarderait l'absorption.

Il est bien connu que l'excrétion urinaire de phosphore diminue lorsque l'apport alimentaire de calcium augmente. Sans exclure totalement une action indirecte du calcium, par l'intermédiaire des parathyroïdes, sur l'excrétion urinaire du phosphore, il semble plus probable que l'excès de calcium agit simplement en favorisant la fixation osseuse du phosphore absorbé. Nous avons vu chez le Porc (GUEGUEn et RÉrat, I963) qu'en présence d'un grand excès de calcium, pratiquement tout le phosphore absorbé était retenu. Quel que soit le mécanisme, encore très discuté, de la fixation du calcium et du phosphore dans le cristal osseux, il semble logique de penser que la présence d'un excès de l'un de ces éléments favorise la fixation de l'autre.

De plus, ne faudrait-il pas limiter cette notion d'équilibre nécessaire en lui associant un "facteur temps "? La simultanéité des arrivées des deux éléments dans le sang nous semble être un facteur important de leur bonne utilisation par l'os. Ainsi s'expliquerait simplement la bonne rétention du phosphore du phosphate disodique, dont le maximum plasmatique coïncide avec celui du calcium du même repas (en majeure partie sous forme de carbonate et de phosphate) et la plus mauvaise rétention du phosphore du son de blé, dont l'absorption est très décalée par rapport à celle du calcium. Ainsi se justifierait également 1'observation souvent faite selon laquelle le phosphore phytique est impropre à assurer la minéralisation de l'os mais permet une bonne croissance corporelle : en fait, la rétention extra-osseuse du phosphore est très souple et ne nécessite pas la présence simultanée de calcium. A l'appui de cette hypothèse, nous pouvons citer les données expérimentales obtenues chez des poulets par HARVILLE et NELSON (I963), montrant que des apports alternés de calcium et de phosphore (décalage de 24 heures) ne permettent pas d'assurer une bonne rétention des deux éléments. Un décalage moins important, tel celui que nous avons enregistré au niveau de l'absorption, devrait avoir également une certaine répercussion sur la rétention du phosphore absorbé.

Cependant, quelques expériences complémentaires effectuées sur des porcs recevant la dose orale de ${ }^{32} \mathrm{PO}_{4} \mathrm{HNa}_{2}$ dans un repas sans calcium (blé seul) nous ont montré que ${ }^{32} \mathrm{P}$ absorbé pouvait être efficacement retenu en 1'absence de toute absorption de calcium alimentaire. Toutefois, ce repas sans calcium ne supprime pas les sécrétions digestives et donc l'importante entrée simultanée dans le sang de calcium endogène réabsorbé. D'autre part, connaissant l'intensité des recyclages du calcium osseux chez le Porc en croissance (BEsançon et al., Ig66) il est logique de penser 
que l'accrétion osseuse du phosphore absorbé sans calcium puisse être accompagnée de l'accrétion de calcium provenant de l'ostéolyse ; toutefois, il en résulterait l'élimination de la quantité équivalente de phosphore détachée simultanément de l'os. Ainsi, une bonne rétention de ${ }^{32} \mathrm{P}$ absorbé ne signifie pas nécessairement que les conditions soient remplies pour la fixation maximale du phosphore circulant.

Cependant, si le calcium provenant directement de l'os peut suffire à assurer une bonne rétention du phosphore alimentaire, en revanche, une rétention maximale ne doit pouvoir se produire qu'en présence d'un excès de calcium circulant. Seul le calcium exogène peut permettre de franchir ce seuil, le calcium libéré de l'os par ostéolyse étant accompagné de la quantité correspondante de phosphore osseux. D'autre part, le rôle joué par l'arrivée de calcium exogène pourrait être accru dans le cas d'une diminution postprandiale de l'ostéolyse, due à une réduction de l'activité des parathyroïdes provoquée par une hypercalcémie passagère.

Nous n'avons fait que soulever une question très complexe mais qui semble justifier des études plus approfondies des rythmes d'entrée du phosphore et du calcium dans le sang, et en particulier des variations éventuelles de l'activité ostéolytique.

Reçu pour publication en décembre 1967.

\section{SUMMARY}

DIGESTIVE UTILIZATION, KINETICS OF ABSORPTION AND EFFICIENCY OF RETENTION OF PHYTIC PHOSPHORUS IN PIGS

The experiment was with $\mathbf{I} 7$ growing pigs kept in metabolism cages. They were given a single dose of ${ }^{32} \mathrm{P}$ in their feed, either as disodium phosphate or as activated wheat bran. Fæces and urine were collected for 6 days after the labelled feed had been given and blood samples were taken for study of the kinetics of intestinal absorption.

True digestive utilization of phosphorus, calculated by the method of comparative balances, reached a mean of 7 1.o per cent for disodium phosphate and 37.5 per cent for wheat bran (table 1 ). When the phytic phosphorus was given in liquid form as a trichloroacetic acid extract of wheat bran there was no improvement in digestive utilization. Hydrolysis of phytates seemed to be mainly in the stomach.

The curves showing changes of radioactivity in plasma (fig. $\mathbf{I}$ ). reached a maximum one hour after the feed had been given for disodium phosphace, and only four hours after for wheat bran. Maximum value for radioactivity in plasma, expressed as percentage of the dose, varied in relation to the rate of absorption and could bot be used as a criterion of the intensity of absorption of the phosphorus.

Efficiency of retention of absorbed phosphorus was less for wheat bran, 6I.3 per cent, than for disodium phosphate, 76.1 per cent. The discussion bears mainly on the interpretation of this difference, which does not seem to be due to the chemical nature of the phosphorus absorbed. A hypothesis is proposed according to which the simultaneous absorption of calcium would favour the maximum retention of absorbed phosphorus in bone.

\section{RÉFÉRENCES BIBLIOGRAPHIQUES}

Ammerman C. B., Forbes R. M., Garrigus U. S., Neumann A. L., Norton H. W., Hatfreld E. E., 1957. Ruminant utilization of inorganic phosphates. J. Anim. Sci., 16, 796-8ro.

Auffray P., Martinet J., RÉrat A., 1967. Quelques aspects du transit gastro-intestinal chez le Porc. Ann. Biol. anim. Bioch. Biophys., 7, 26I-279.

Avioli L. V., McDonald J. E., Singer R. A., Henneman P. H., ig65. A new oral isotopic test of calcium absorption. J. Clin. Invest., 44, I 28-г 39 . 
Besançon P., Gú́gues L., RÉRat A., 1966. Échanges et vitesse d'incorporation du calcium dans le squelette du Porc en croissance. C. R. Acad. Sci. Paris, 263, I I34-1 137.

Caniggia A., Gennari C., 1965. L'assorbimento intestinale del radiocalcio $\left({ }^{45} \mathrm{Ca}-{ }^{47} \mathrm{Ca}\right)$ nell'Uomo adulto e nel vecchio in condizioni normali e patologiche. G. Geront., 13, 762-765.

Caniggia A., Gennari C., Bencini M., Palazzuoli V., Cesari L.. ig66. L'assorbimento intestinale del radiofosfato $\left(\mathrm{Na}_{2} \mathrm{H}^{32} \mathrm{PO}_{4}\right)$ nell'Uomo. Boll. Soc. ital. Biol. Sper., 42, 583-587,

Comar C. L., Monroe R. A., Visek W. J., Hansard S. L., I953. Comparison of two isotopic methods for determination of endogenous fecal calcium. J. Nutr., 50, 459-467.

Gú́guen L., Rérat A., 1965. Contribution à l'étude de l'absorption intestinale et du mode d'excrétion du phosphore chez le Porc. C. R. Acad. Sci. Paris, 260, 5112-5I 5 .

GuÉguen L., Rérat A., 1967. Cinétique de l'absorption intestinale du phosphore chez le Porc. Ann. Biol. anim. Biochim. Biophys., 7, 39-46.

HaRville D. A., Nelson T. S., I963. The effect of alternate intake on the utilization of calcium or phosphorus by chicks (Abstr.). Poultry Sci., 42, 1275-1276.

Hill R., TYlER C., I954a. The effect of increasing acidity on the solubility of calcium, magnesium and phosphorus in certain cereals and pure salts. J. Agric. Sci., 44, 283-305.

Hill R., Tyler C., I954 b. The influence of time, temperature, $\mathrm{pH}$ and calcium carbonate on the activity of the phytase of certain cereals. J. Agric. Sci., 44, 306-310.

Holt R., 1955. Studies on dried peas. I. The determination of phytate phosphorus. J. Sci. Food Agric., 6, I36-I42.

MOORE J. H., Tyler C., I955 $a$. Studies on the intestinal absorption and excretion of calcium and phosphorus in the pig. I. A critical study of the Bergeim technique for investigating the intestinal absorption and excretion of calcium and phosphorus. Brit. J. Nutr., 9, 63-8r.

Moore J. H., Tyler C., r955 b. Studies on the intestinal absorption and excretion of calcium and phosphorus in the pig. II. The intestinal absorption of radioactive calcium and phosphorus. Brit. $J . N u t r ., 9$, 81-93.

Nelson T.S., 1967. The utilization of phytate phosphorus by poultry. A review. Poullry Sci., 46, 862-87r,

SсHонд А. T., 1939. Mineral Metabolism. 187. Rheinhold corp., New York.

SINGSEN E. P., I948. The phosphorus requirement of the chicken with special reference to the availability of phytin P. Storrs Agric. Exp. Sta. Bull., 260.

Singsen E. P., Matterson L. D., Koseff A., I950. Phosphorus in poultry nutrition. 4. Radioactive phosphorus as a tracer in studying the metabolism of phytin in turkey poult. Poultry Sci., 29, 635-639.

Temperton H., Cassidy J., rg64a. Phosphorus requirements of poultry. I. The utilization of phytin phosphorus by the chick as indicated by balance experiments. Brit. Poulliry Sci., 5, 75-80.

Temperton H., CAssidy J., $1964 b$. Phosphorus requirements of poultry. II. The utilization of phytin phosphorus by the chick for growth and bone formation. Brit. Poultry Sci., 5, 8I-86.

Waldroup P. W., Ammerman C. B., Harms R. H., 1965. The availability of phytic acid phosphorus for chicks. 4. The availability of natural plant phosphorus. Poultry Sci., 44, 880-886.

Waldroup P. W., Simpson C. F., Damron B. L., Harms R. H., ig67. The effectiveness of plant and inorganic phosphorus in supporting egg production in hens and hatchability and bone development in chick embryos. Poultry Sci., 46, 659-663. 